\title{
SOME WEAK FRAGMENTS OF MARTIN'S AXIOM RELATED TO THE RECTANGLE REFINING PROPERTY
}

\author{
TERUYUKI YORIOKA
}

\begin{abstract}
We introduce the anti-rectangle refining property for forcing notions and investigate fragments of Martin's axiom for $\aleph_{1}$ dense sets related to the anti-rectangle refining property, which is close to some fragment of Martin's axiom for $\aleph_{1}$ dense sets related to the rectangle refining property, and prove that they are really weaker fragments.
\end{abstract}

\section{INTRODUCTION}

In [12], Larson-Todorčević introduced the rectangle refining property of partition on $\left[\omega_{1}\right]^{2}$, which is stronger than ccc (as the property for partitions, defined in e.g. $[16])$, and the fragment $\mathcal{K}_{2}(\mathrm{rec})$ of Martin's axiom for $\aleph_{1}$ dense sets, denoted by $M A_{\aleph_{1}}$, to show the consistency of Katětov's statement. In this note, we analyze relationships of $\mathcal{K}_{2}(\mathrm{rec})$ and other fragments of $\mathrm{MA}_{\aleph_{1}}$.

In this note, we introduce some property which is mutually had by both an Aronszajn tree and an $\left(\omega_{1}, \omega_{1}\right)$-gap, which will be called the anti-rectangle refining property. In [2], Abraham-Todorčević pointed out the similarity between Aronszajn trees and $\left(\omega_{1}, \omega_{1}\right)$-gaps. The anti-rectangle refining property defined below is derived from its similarity. As a forcing notion, an Aronszajn tree has the antirectangle refining property, and a forcing notion freezing an $\left(\omega_{1}, \omega_{1}\right)$-gap also has the anti-rectangle refining property.

In 1980's, Todorčević investigated several fragments of $\mathrm{MA}_{\aleph_{1}}$. One of them is the statement $\mathcal{K}_{n}$, for a natural number $n$ larger than 1 , that for every ccc forcing notion $\mathbb{P}$, all uncountable subsets of $\mathbb{P}$ have uncountable $n$-linked subsets. We note easily that every $\mathcal{K}_{n}$ is weaker than $\mathrm{MA}_{\aleph_{1}}$ and $\mathcal{K}_{n+1}$ implies $\mathcal{K}_{n}$. Todorčević also defined another fragment $\mathcal{C}^{2}$ of $\mathrm{MA}_{\aleph_{1}}$, which is the statement that any product of ccc forcing notions is still ccc. We also notice that $\mathcal{K}_{2}$ implies $\mathcal{C}^{2}$. However it is still unknown that the weakest fragment $\mathcal{C}^{2}$ in fragments mentioned above is essentially weaker than $M A_{\aleph_{1}}$, that is, it is still open whether it is consistent that $\mathcal{C}^{2}$ holds but $M A_{\aleph_{1}}$ fails. In this note, we introduce fragments of $M A_{\aleph_{1}}$ on the anti-rectangle refining property, $\mathrm{MA}_{\aleph_{1}}(a(\operatorname{arec})), \mathcal{K}_{2}(a(\operatorname{arec}))$ and $\neg \mathcal{C}(\operatorname{arec})$, and prove that they are essentially weaker than $\mathrm{MA}_{\aleph_{1}}$, more precisely, we show that it is consistent that $\operatorname{MA}(a(\operatorname{arec}))$ holds but $\mathcal{C}^{2}$ fails.

In section 2 , we define the anti-rectangle refining property and see a basic fact. In section 3 , we give examples related to the anti-rectangle refining property. In

Department of Mathematics, Shizuoka University, Ohya 836, Shizuoka, 422-8059, JAPAN

Tel.: +81-54-238-4721

styorio@ipc.shizuoka.ac

Partially supported by Grant-in-aids for Scientific Research No.16340022 and No.18840022. 
section 4, we investigate fragments of $\mathrm{MA}_{\aleph_{1}}$ on the anti-rectangle refining property and show that our fragments are essentially weaker than $\mathrm{MA}_{\aleph_{1}}$.

\section{The Definition of the Anti-ReCtangle REFining PROPERTy}

A forcing notion has the countable chain condition (ccc) if it does not have an uncountable antichain (an uncountable pairwise incompatible set of conditions). We introduce a new chain condition which can be considered as the variation of the property K.

Definition 2.1. - (Knaster, [8]) A forcing notion $\mathbb{P}$ has property $K$ if for every $I \in[\mathbb{P}]^{\aleph_{1}}$, there is a refinement $I^{\prime} \in[I]^{\aleph_{1}}$ such that $p$ and $q$ are compatible in $\mathbb{P}$ (i.e. there exists $r \in \mathbb{P}$ such that both $r \leq_{\mathbb{P}} p$ and $r \leq_{\mathbb{P}} q$ hold, denoted by $\left.p \not_{\mathbb{P}} q\right)$ for every $p$ and $q$ in $I^{\prime}$.

- A forcing notion $\mathbb{P}$ has the anti-rectangle refining property if it is uncountable and for every $I$ and $J$ in $[\mathbb{P}]^{\aleph_{1}}$, there are refinements $I^{\prime} \in[I]^{\aleph_{1}}$ and $J^{\prime} \in[J]^{\aleph_{1}}$ such that $p$ and $q$ are incompatible in $\mathbb{P}$ (denoted by $p \perp_{\mathbb{P}} q$ ) for every $p \in I^{\prime}$ and $q \in J^{\prime}$.

For a forcing notion $\mathbb{P}$, we define another forcing notion $a(\mathbb{P})$ which consists of finite antichains in $\mathbb{P}$, ordered by reverse inclusion. If $a(\mathbb{P})$ has the ccc, then by the genericity, $a(\mathbb{P})$ can add an uncountable antichain in $\mathbb{P}$. So it follows from the next lemma that if $\mathbb{P}$ has the ccc and the anti-rectangle refining property, then $a(\mathbb{P})$ can destroy the ccc property of $\mathbb{P}$.

Proposition 2.2. If a forcing notion $\mathbb{P}$ has the anti-rectangle refining property, then for every $I$ and $J$ in the set $[a(\mathbb{P})]^{\aleph_{1}}$ with $I \cup J$ pairwise disjoint, i.e. $\sigma \cap \tau=\emptyset$ for any distinct $\sigma$ and $\tau$ in $I \cup J$, there are refinements $I^{\prime} \in[I]^{\aleph_{1}}$ and $J^{\prime} \in[J]^{\aleph_{1}}$ such that for any $\sigma \in I^{\prime}$ and $\tau \in J^{\prime}, \sigma \not \perp_{a(\mathbb{P})} \tau$, i.e. $\sigma \cup \tau \in a(\mathbb{P})$.

This property is very similar to the rectangle refining property.

Definition 2.3 (Larson-Todorčević, [11, Definition 4.1.]). A partition $\left[\omega_{1}\right]^{2}=K_{0} \cup$ $K_{1}$ satisfies the rectangle refining property if for every $I$ and $J$ in the set $\left[\omega_{1}\right]^{\aleph_{1}}$, there are refinements $I^{\prime} \in[I]^{\aleph_{1}}$ and $J^{\prime} \in[J]^{\aleph_{1}}$ such that

$$
\left\{\{\alpha, \beta\} ; \alpha \in I^{\prime} \& \beta \in J^{\prime} \& \alpha<\beta\right\} \subseteq K_{0} .
$$

This is the reason why I call the anti-rectangle refining property.

We note that if a partition $\left[\omega_{1}\right]^{2}=K_{0} \cup K_{1}$ satisfies the rectangle refining property, then for every $I$ and $J$ in $\left[\omega_{1}\right]^{\aleph_{1}}$, there are refinements $I^{\prime} \in[I]^{\aleph_{1}}$ and $J^{\prime} \in[J]^{\aleph_{1}}$ such that $\left(I^{\prime}\right.$ and $J^{\prime}$ are disjoint and)

$$
\left\{\{\alpha, \beta\} ; \alpha \in I^{\prime} \& \beta \in J^{\prime}\right\} \subseteq K_{0} .
$$

Moreover we should note that this property is stronger than the ccc, so if $\mathbb{P}$ has the anti-rectangle refining property, then $a(\mathbb{P})$ has the ccc.

Proof of Proposition 2.2. Assume that $\mathbb{P}$ has the anti-rectangle refining property and let $I$ and $J$ be uncountable subsets of $a(\mathbb{P})$ such that $I \cup J$ is pairwise disjoint. By shrinking $I$ and $J$ respectively if need, we may assume that each element of $I$ has size $m$ and each element of $J$ has size $n$. Applying the anti-rectangle refining property $m n$ times, we can find uncountable subsets $I^{\prime}$ and $J^{\prime}$ of $I$ and $J$ respectively such that for every $\sigma \in I^{\prime}$ and $\tau \in J^{\prime}$, the $i$-th condition in $\sigma$ and the $j$-th condition 
in $\tau$ are incompatible in $\mathbb{P}$ for each $i \in m$ and $j \in n$. Then every element of $I^{\prime}$ and every element of $J^{\prime}$ are compatible in $a(\mathbb{P})$.

We notice that $a(\mathbb{P})$ may contain an atom, that is, there are no strict extension of it. In $a(\mathbb{P})$, an atom means a finite maximal antichain in $\mathbb{P}$. We consider $a^{\prime}(\mathbb{P})$ which consists of conditions in $a(\mathbb{P})$ which are not atoms. Then we can show by the same argument in the proof of Proposition 2.2, if $\mathbb{P}$ has the anti-rectangle refining property, then $a^{\prime}(\mathbb{P})$ also has the rectangle refining property. (Let $I$ and $J$ be uncountable subsets of $a^{\prime}(\mathbb{P})$ such that each element of $I$ has size $m$ and each element of $J$ has size $n$. For each $\sigma \in I$, we take any $p_{\sigma} \in \mathbb{P}$ such that $\sigma \cup\left\{p_{\sigma}\right\}$ is also a condition in $a(\mathbb{P})$. ( $p_{\sigma}$ is a dummy condition.) Applying the anti-rectangle refining property $(m n+n)$ times, we can find uncountable subsets $I^{\prime}$ and $J^{\prime}$ of $I$ and $J$ respectively such that for every $\sigma \in I^{\prime}$ and $\tau \in J^{\prime}$, any condition in $\sigma \cup\left\{p_{\sigma}\right\}$ and any condition in $\tau$ are incompatible in $\mathbb{P}$. Then every element of $I^{\prime}$ and every element of $J^{\prime}$ are compatible in $a^{\prime}(\mathbb{P})$.) We note that if $\mathbb{P}$ has the anti-rectangle refining property and for every countable subset $I$ of $\mathbb{P}$ and $\sigma \in a^{\prime}(\mathbb{P})$, there exists an extension $\tau$ of $\sigma$ in $a^{\prime}(\mathbb{P})$ which is not a subset of $I$, then

$$
\Vdash_{a^{\prime}(\mathbb{P})} \text { " } \bigcup \dot{G} \text { is an uncountable antichain ". }
$$

For example, if a forcing notion $\mathbb{P}$ with the anti-rectangle refining property satisfies that for every condition $p$ in $\mathbb{P}$, the set of predecessors of $p$, i.e. $\{q \in \mathbb{P} ; q \leq \mathbb{P} p\}$, is uncountable, then $a^{\prime}(\mathbb{P})$ has this property.

\section{EXAMPLES}

3.1. $\omega_{1}$-trees. A tree is an ordered structure $\left\langle T,<_{T}\right\rangle$ such that for each $t \in T$, the predecessors $\left\{s \in T ; s<_{T} t\right\}$ of $t$ is well ordered with respect to $<_{T}$. We note that in a tree-order, the compatibility is same to the comparability. A tree $T$ is an $\omega_{1}$-tree if $T$ has height $\omega_{1}$ and every level of $T$ is countable. An $\omega_{1}$-tree is Aronszajn if it does not have uncountable branches, and is Suslin if it does not have both uncountable branches and uncountable pairwise incomparable subsets. So a Suslin tree is an Aronszajn tree which has the countable chain condition as a forcing notion (with the reverse order of the original order). It is well known that there exists an Aronszajn tree (under ZFC) and Suslin's hypothesis is equivalent to the statement that there are no Suslin trees.

Proposition 3.1. An Aronszajn tree has the anti-rectangle refining property.

Proof. Suppose that $T$ is an Aronszajn tree.

Claim 3.2. If $I$ is an uncountable subset of $T$, then there are $s_{0}$ and $s_{1}$ such that $s_{0}$ and $s_{1}$ are incomparable in $T$ and the sets $\left\{u \in I ; s_{0}<_{T} u\right\}$ and $\left\{u \in I ; s_{1}<_{T} u\right\}$ are both uncountable.

Proof of Claim 3.2. Assume not. Then for each $\alpha \in \omega_{1}$, there exists $t_{\alpha}$ in an $\alpha$-th level of $T$ such that the set $\left\{u \in I ; t_{\alpha}<_{T} u\right\}$ is uncountable. By our assumption, the family $\left\{t_{\alpha} ; \alpha \in \omega_{1}\right\}$ is pairwise comparable, that is, a chain through $T$, from which it follows that $T$ is not Aronszajn.

Let $I$ and $J$ be in $[T]^{\aleph_{1}}$. By the above claim, we can find $s_{0}, s_{1}, t_{0}$ and $t_{1}$ in $T$ such that $s_{0}$ and $s_{1}$, and $t_{0}$ and $t_{1}$ are incomparable in $T$ respectively and the 
sets $\left\{u \in I ; s_{i}<_{T} u\right\}$ and $\left\{u \in J ; t_{j}<_{T} u\right\}$ are uncountable for each $i, j \in\{0,1\}$. Let $i, j \in\{0,1\}$ be such that $s_{i}$ and $t_{j}$ are incomparable in $T$ and then let $I^{\prime}:=$ $\left\{u \in I ; s_{i}<_{T} u\right\}$ and $J^{\prime}:=\left\{u \in J ; t_{j}<_{T} u\right\}$. Then every node in $I^{\prime}$ is incomparable with every node in $J^{\prime}$.

This proposition and Proposition 2.2 follows that, if $T$ is an Aronszajn tree, then $a(T)$ has the ccc, which is essentially due to Baumgartner in [4]. (However, this proof is much simpler than Baumgartner's one.)

From [10, Theorem 11.], it follows that a forcing notion with property $\mathrm{K}$ cannot add an uncountable antichain in a Suslin tree. So if $T$ is a Suslin tree, $a(T)$ (in fact $T$ itself) does not have property $\mathrm{K}$.

3.2. $\left(\omega_{1}, \omega_{1}\right)$-pregaps. An $\left(\omega_{1}, \omega_{1}\right)$-pregap is a sequence $\left\langle a_{\alpha}, b_{\alpha} ; \alpha \in \omega_{1}\right\rangle$ of reals (which means sets of natural numbers) such that

- for every $\alpha<\beta$ in $\omega_{1}, a_{\alpha}$ and $b_{\alpha}$ are almost contained in $a_{\beta}$ and $b_{\beta}$ respectively, that is, both $a_{\alpha} \backslash a_{\beta}$ and $b_{\alpha} \backslash b_{\beta}$ are finite (and then we denote $a_{\alpha} \subseteq^{*} a_{\beta}$ and $\left.b_{\alpha} \subseteq^{*} b_{\beta}\right)$,

- for every $\alpha \neq \beta$ in $\omega_{1}, a_{\alpha}$ and $b_{\beta}$ are almost disjoint, that is, the set $a_{\alpha} \cap b_{\beta}$ is finite.

An $\left(\omega_{1}, \omega_{1}\right)$-pregap $\left\langle a_{\alpha}, b_{\alpha} ; \alpha \in \omega_{1}\right\rangle$ is called a gap if there is no set $c$ of natural numbers such that for all $\alpha \in \omega_{1}, a_{\alpha}$ is almost contained in $c$ and $b_{\alpha}$ is almost disjoint from $c$. If this $c$ exists, it is called an interpolation of $(\mathcal{A}, \mathcal{B})$. For an $\left(\omega_{1}, \omega_{1}\right)$-pregap $(\mathcal{A}, \mathcal{B})=\left\langle a_{\alpha}, b_{\alpha} ; \alpha \in \omega_{1}\right\rangle$ with the set $a_{\alpha} \cap b_{\alpha}$ empty for every $\alpha \in \omega_{1}$, we have the following characterization of being a gap (due to Kunen and Todorčević). $(\mathcal{A}, \mathcal{B})$ is a gap iff it has the following property (f) :

For every uncountable set $X$ of countable ordinals, there exists different ordinals $\alpha$ and $\beta$ in $X$ such that

$$
\left(a_{\alpha} \cap b_{\beta}\right) \cup\left(a_{\beta} \cap b_{\alpha}\right) \neq \emptyset .
$$

An $\left(\omega_{1}, \omega_{1}\right)$-pregap $\left\langle a_{\alpha}, b_{\alpha} ; \alpha \in \omega_{1}\right\rangle$ is called destructible if it is not a gap in some forcing extension not collapsing cardinals. In this paper, a destructible gap means an $\left(\omega_{1}, \omega_{1}\right)$-pregap which forms a gap and is destructible. For an $\left(\omega_{1}, \omega_{1}\right)$-pregap $(\mathcal{A}, \mathcal{B})=\left\langle a_{\alpha}, b_{\alpha} ; \alpha \in \omega_{1}\right\rangle$ with the set $a_{\alpha} \cap b_{\alpha}$ empty for every $\alpha \in \omega_{1}$, we have the following characterization of destructibility (also due to Kunen and Todorčević). $(\mathcal{A}, \mathcal{B})$ is destructible iff it has the following property $(\mathrm{s})$ :

For every uncountable set $X$ of countable ordinals, there exists different ordinals $\alpha$ and $\beta$ in $X$ such that

$$
\left(a_{\alpha} \cap b_{\beta}\right) \cup\left(a_{\beta} \cap b_{\alpha}\right)=\emptyset .
$$

For each $\left(\omega_{1}, \omega_{1}\right)$-pregap $(\mathcal{A}, \mathcal{B})=\left\langle a_{\alpha}, b_{\alpha} ; \alpha \in \omega_{1}\right\rangle$ with the set $a_{\alpha} \cap b_{\alpha}$ empty for every $\alpha \in \omega_{1}$, we can define two types of forcing notions (see e.g. [5], [9], [13], $[16])$ :

- $\mathcal{F}(\mathcal{A}, \mathcal{B}):=\left\{\sigma \in\left[\omega_{1}\right]^{<\omega} ; \forall \alpha \neq \beta\right.$ in $\left.\sigma,\left(a_{\alpha} \cap b_{\beta}\right) \cup\left(a_{\beta} \cap b_{\alpha}\right) \neq \emptyset\right\}$, ordered by reverse inclusion.

- $\mathcal{S}(\mathcal{A}, \mathcal{B}):=\left\{\sigma \in\left[\omega_{1}\right]^{<\omega} ; \forall \alpha \neq \beta\right.$ in $\left.\sigma,\left(a_{\alpha} \cap b_{\beta}\right) \cup\left(a_{\beta} \cap b_{\alpha}\right)=\emptyset\right\}$, ordered by reverse inclusion. 
We note that $\mathcal{F}(\mathcal{A}, \mathcal{B})$ generically adds a counterexample of property (s) and and $\mathcal{S}(\mathcal{A}, \mathcal{B})$ generically adds a counterexample of property (f). The following characterizations of being a gap and destructibility has been proved (also due to Kunen and Todorčević):

- $(\mathcal{A}, \mathcal{B})$ is a gap iff $\mathcal{F}(\mathcal{A}, \mathcal{B})$ has the countable chain condition.

- $(\mathcal{A}, \mathcal{B})$ is destructible iff $\mathcal{S}(\mathcal{A}, \mathcal{B})$ has the countable chain condition.

So $(\mathcal{A}, \mathcal{B})$ is a destructible gap iff both $\mathcal{F}(\mathcal{A}, \mathcal{B})$ and $\mathcal{S}(\mathcal{A}, \mathcal{B})$ have the countable chain condition.

Proposition $3.3\left(\left[7\right.\right.$, Lemma B.1]). Let $(\mathcal{A}, \mathcal{B})=\left\langle a_{\alpha}, b_{\alpha} ; \alpha \in \omega_{1}\right\rangle$ be an $\left(\omega_{1}, \omega_{1}\right)$ gap. Then for every $I$ and $J$ in the set $\left[\omega_{1}\right]^{\aleph_{1}}$, there are refinements $I^{\prime} \in[I]^{\aleph_{1}}$ and $J^{\prime} \in[J]^{\aleph_{1}}$ such that for every $\alpha \in I^{\prime}$ and $\beta \in J^{\prime}, a_{\alpha} \cap b_{\beta} \neq \emptyset$.

Proof. For each $\alpha \in \omega_{1}$, there is a natural number $n_{\alpha}$ such that both sets $\left\{\xi \in \omega_{1} ; a_{\alpha} \backslash n_{\alpha} \subseteq a_{\xi}\right\}$ and $\left\{\eta \in \omega_{1} ; b_{\alpha} \backslash n_{\alpha} \subseteq b_{\eta}\right\}$ are uncountable. We note that the set

$$
\bigcup_{\xi \in I}\left(a_{\xi} \backslash n_{\xi}\right) \cap \bigcup_{\eta \in J}\left(b_{\eta} \backslash n_{\eta}\right)
$$

is not empty because the pregap

$$
\left\langle a_{\xi} \backslash n_{\xi}, b_{\eta} \backslash n_{\eta} ; \xi \in I, \eta \in J\right\rangle
$$

is equivalent to the original one and so is a gap. We take $\alpha \in I, \beta \in J$ and $k \in \omega$ such that $k$ is in the set $\left(a_{\alpha} \backslash n_{\alpha}\right) \cap\left(b_{\beta} \backslash n_{\beta}\right)$. Let $I^{\prime}:=\left\{\xi \in I ; a_{\alpha} \backslash n_{\alpha} \subseteq a_{\xi}\right\}$ and $J^{\prime}:=\left\{\eta \in J ; b_{\beta} \backslash n_{\beta} \subseteq b_{\eta}\right\}$ which are as desired.

Corollary 3.4. $\mathcal{S}(\mathcal{A}, \mathcal{B})$ has the anti-rectangle refining property.

Proof. This can be proved as arguments in the proof of Proposition 2.2 using the above proposition.

By Corollary 3.4, $a(\mathcal{S}(\mathcal{A}, \mathcal{B}))$ has the ccc, and hence it freezes $(\mathcal{A}, \mathcal{B})$, that is, it forces $(\mathcal{A}, \mathcal{B})$ to be indestructible. In fact, if an $\left(\omega, \omega_{1}\right)$-gap $(\mathcal{A}, \mathcal{B})$ with the set $a_{\alpha} \cap b_{\alpha}$ empty for every $\alpha \in \omega_{1}$ is closed under finite modifications (in [17], it is said that $(\mathcal{A}, \mathcal{B})$ admits closed under finite changes $)$, then $a(\mathcal{S}(\mathcal{A}, \mathcal{B}))$ is forcingequivalent to $\mathcal{F}(\mathcal{A}, \mathcal{B})$.

As in the case of $\omega_{1}$-trees, a forcing notion with property $\mathrm{K}$ cannot destroy a destructible gap, i.e. if $\mathbb{P}$ is a forcing notion with property $K$ and $(\mathcal{A}, \mathcal{B})$ is an $\left(\omega_{1}, \omega_{1}\right)$-pregap,

- and if $(\mathcal{A}, \mathcal{B})$ is a gap, then it is still a gap in the extension by $\mathbb{P}$,

- and if $(\mathcal{A}, \mathcal{B})$ is destructible, then it is still destructible in the extension by $\mathbb{P}$.

So we note that if $(\mathcal{A}, \mathcal{B})$ is a destructible gap, then both $\mathcal{S}(\mathcal{A}, \mathcal{B})$ and $\mathcal{F}(\mathcal{A}, \mathcal{B})$ do not have property $\mathrm{K}$.

3.3. The bounding number $\mathfrak{b}$. For functions $f$ and $g$ in $\omega^{\omega}$, we define $f \leq g$ when $f(i) \leq g(i)$ for all $i \in \omega$, and define $f \leq^{*} g$ when $f(i) \leq g(i)$ for all but finite many $i \in \omega$. The bounding number $\mathfrak{b}$ is the smallest size of an unbounded family in the structure $\left\langle\omega^{\omega}, \leq^{*}\right\rangle$. For a family $\mathcal{F}$ of functions in $\omega^{\omega}$, we define a partition $[\mathcal{F}]^{2}=K_{0}^{\mathcal{F}} \cup K_{1}^{\mathcal{F}}$ such that for distinct members $f$ and $g$ in $\mathcal{F}$,

$$
\{f, g\} \in K_{0}^{\mathcal{F}}: \Longleftrightarrow f \not \leq g \text { and } g \not \leq f \text {. }
$$


Proposition 3.5. Assume that $\mathcal{F}=\left\{f_{\xi} ; \xi \in \omega_{1}\right\}$ is a $\leq^{*}$-well ordered unbounded family in $\left\langle\omega^{\omega}, \leq^{*}\right\rangle$.

For every $I$ and $J$ in the set $\left[\omega_{1}\right]^{\aleph_{1}}$, there are refinements $I^{\prime \prime} \in[I]^{\aleph_{1}}$ and $J^{\prime \prime} \in$ $[J]^{\aleph_{1}}$ and $k$ and $l$ in $\omega$ such that for every $\xi \in I^{\prime \prime}$ and $\eta \in J^{\prime \prime}, f_{\xi}(k)<f_{\eta}(k)$ and $f_{\eta}(l)<f_{\xi}(l)$.

Proof. Let $I$ and $J$ be in $\left[\omega_{1}\right]^{\aleph_{1}}$. We take any injection $\pi$ from $I$ into $J$ such that for every $\xi \in I, f_{\pi(\xi)} \mathbb{Z}^{*} f_{\xi}$. This can be done by our assumption. So for each $\xi \in I$, there exists $k_{\xi} \in \omega$ such that $f_{\xi}\left(k_{\xi}\right)<f_{\pi(\xi)}\left(k_{\xi}\right)$. There exist $k, m_{0}, m_{1} \in \omega$ and $I^{\prime} \in[I]^{\aleph_{1}}$ such that $k_{\xi}=k, f_{\xi}(k)=m_{0}$ and $f_{\pi(\xi)}(k)=m_{1}$ for every $\xi \in I^{\prime}$ and then let $J^{\prime}:=\left\{\pi(\xi) ; \xi \in I^{\prime}\right\}$.

Now, we repeat a same argument with the role of $I$ and $J$ exchanged. We take any injection $\pi^{\prime}$ from $J^{\prime}$ into $I^{\prime}$ such that $f_{\pi^{\prime}(\xi)} \mathbb{Z}^{*} f_{\xi}$. Then there exist $l, n_{0}, n_{1} \in \omega$ and $J^{\prime \prime} \in\left[J^{\prime}\right]^{\aleph_{1}}$ such that for every $\xi \in J^{\prime \prime}, f_{\xi}(l)=n_{0}<n_{1}=f_{\pi^{\prime}(\xi)}(l)$. Let $I^{\prime \prime}:=\left\{\pi^{\prime}(\xi) ; \xi \in J^{\prime}\right\}$. Then $I^{\prime \prime}$ and $J^{\prime \prime}$ are as desired.

Corollary 3.6. The partition $[\mathcal{F}]^{2}=K_{0}^{\mathcal{F}} \cup K_{1}^{\mathcal{F}}$ has the rectangle refining property.

Proof. This can also be proved as arguments in the proof of Proposition 2.2 using the above proposition.

\section{Fragments of Martin's Axiom}

4.1. Definitions and implications. In this subsection, we see general results on the anti-rectangle refining property and relationships between fragments of Martin's axiom.

Definition 4.1. - (E.g. $[6,(16.4)]$ ) Let $\kappa$ be a cardinal less than the continuum and $\mathcal{P}$ a set of ccc forcing notions. $\operatorname{MA}_{\kappa}(\mathcal{P})$ denotes the statement that for any $\mathbb{P} \in \mathcal{P}$ and a family $\mathcal{D}$ of dense subsets of $\mathbb{P}$ with $|\mathcal{D}| \leq \kappa$, there exists a filter $G$ on $\mathbb{P}$ such that $G \cap D \neq \emptyset$ for every $D \in \mathcal{D}$. $\operatorname{MA}(\mathcal{P})$ denotes the statement that all $\mathrm{MA}_{\kappa}(\mathcal{P})$ holds for every cardinal $\kappa$ less than the continuum.

$\mathrm{MA}_{\aleph_{1}}$ is $\mathrm{MA}_{\aleph_{1}}(\mathrm{CCC})$ where $\mathrm{CCC}$ is the set of ccc forcing notions, $\mathrm{MA}_{\aleph_{1}}$ (propertyK) is $\mathrm{MA}_{\aleph_{1}}$ for forcing notions with property $K$ (in e.g. [10]), and $\mathrm{MA}_{\aleph_{1}}(a(\operatorname{arec})$ ) is $\mathrm{MA}_{\aleph_{1}}(\mathcal{P})$ where $\mathcal{P}$ is the set of forcing notions a $(\mathbb{P})$ for $\mathbb{P}$ with the antirectangle refining property.

- (E.g. $[15, \S 2]) \mathcal{K}_{2}$ denotes the statement that every ccc forcing notion has property K. (For more on this definition, see the above of Proposition 4.5.)

- $([12, \S 4]) \mathcal{K}_{2}(\mathrm{rec})$ denotes the statement that every partition of $\left[\omega_{1}\right]^{2}$ satisfying the rectangle refining property has an uncountable homogeneous set.

- $\mathcal{K}_{2}(a(\operatorname{arec}))$ denotes the statement that for every forcing notion $\mathbb{P}$ with the anti-rectangle refining property, a $(\mathbb{P})$ has property $K$.

- (E.g. [14] or $[11, \S 4]) \mathcal{C}^{2}$ denotes the statement that the product $\mathbb{P} \times \mathbb{Q}$ has the countable chain condition if both forcing notions $\mathbb{P}$ and $\mathbb{Q}$ have the countable chain condition.

- $\neg \mathcal{C}($ arec $)$ denotes the statement that every forcing notion with the antirectangle refining property have an uncountable antichain, that is, it does not have the ccc.

In the next proposition, We list three applications of fragments of $\mathrm{MA}_{\aleph_{1}}$ about examples we saw above. All of them are essentially due to Todorčević. 
Proposition 4.2. $\quad(1) \neg \mathcal{C}($ arec $)$ implies Suslin's hypothesis.

(2) $\neg \mathcal{C}(\operatorname{arec})$ implies that all $\left(\omega_{1}, \omega_{1}\right)$-gaps are indestructible.

(3) $\mathcal{K}_{2}(\mathrm{rec})$ implies $\mathfrak{b}>\aleph_{1}$.

Proof. The first and second statements follows from subsections 3.1 and 3.2.

The third statement follows from Proposition 3.5 and the following lemma [16, 0.7. Lemma]:

If $\mathcal{F}$ is an unbounded family in $\left\langle\omega^{\omega}, \leq^{*}\right\rangle$ and $F$ is a cofinal subset of finite power $\mathcal{F}^{k}$ for some $k \in \omega$, then there exist $x$ and $y$ in $F$ such that $x(i) \leq y(i)$ for every $i \in k$.

It is well known that $\mathrm{MA}_{\aleph_{1}}$ implies $\mathcal{K}_{2}$, and $\mathcal{K}_{2}$ implies $\mathcal{C}^{2}$. It is easy to see that $\mathrm{MA}_{\aleph_{1}}$ implies $\mathrm{MA}_{\aleph_{1}}(a(\operatorname{arec}))$ by definitions, and $\mathrm{MA}_{\aleph_{1}}(a(\operatorname{arec}))$ implies $\mathcal{K}_{2}(a(\operatorname{arec}))$ and $\mathcal{K}_{2}$ implies $\mathcal{K}_{2}(a(\operatorname{arec}))$ by Proposition 2.2 . We will prove that $\neg \mathcal{C}(\operatorname{arec})$ is derived from each of statements $\mathcal{C}^{2}$ and $\mathcal{K}_{2}(a(\operatorname{arec}))$. (A diagram of implications of them is at the end of this section.)

Proposition 4.3. $\mathcal{C}^{2}$ implies $\neg \mathcal{C}($ arec $)$.

Proof. If $\mathbb{P}$ has both the countable chain condition and the anti-rectangle refining property (hence $\mathbb{P}$ is uncountable), then by Proposition 2.2, $a(\mathbb{P})$ is ccc. But the family $\{\langle p,\{p\}\rangle ; p \in \mathbb{P}\}$ of conditions in the product $\mathbb{P} \times a(\mathbb{P})$ forms an antichain in $\mathbb{P} \times a(\mathbb{P})$, hence $\mathbb{P} \times a(\mathbb{P})$ does not have the countable chain condition.

Proposition 4.4. $\mathcal{K}_{2}(a(\operatorname{arec}))$ implies $\neg \mathcal{C}($ arec $)$.

Proof. Let $\mathbb{P}$ be a forcing notion with the anti-rectangle refining property. Then by $\mathcal{K}_{2}(a(\operatorname{arec}))$, there exists an uncountable subset $I$ of $a(\mathbb{P})$ which is pairwise compatible in $a(\mathbb{P})$. That is, for conditions $\sigma$ and $\tau$ in $I, \sigma \cup \tau$ is still a finite antichain in $\mathbb{P}$. Then $\bigcup I$ is an uncountable antichain in $\mathbb{P}$.

It is unknown whether $\neg \mathcal{C}(\operatorname{arec})$ implies $\mathcal{K}_{2}(a(\operatorname{arec}))$. However the next proposition says that $\mathcal{K}(a(\operatorname{arec}))$ and $\neg \mathcal{C}($ arec $)$ are very close statements.

The next proposition has an another viewpoint. $\mathcal{K}_{2}$ is defined by Todorčević in several papers. In [11, Definition 4.9] and $[15, \S 2], \mathcal{K}_{2}$ is defined as above, however in $[12, \S 4]$ and $[16, \S 7], \mathcal{K}_{2}$ is defined by the following statement, denoted by $\mathcal{K}_{2}^{\prime}$ in this note, that

every ccc partition on $\left[\omega_{1}\right]^{2}$ has an uncountable homogeneous set.

(In $[16, \S 7]$, Todorčević said that both statements are very close.) It is easy to show that $\mathcal{K}_{2}$ implies $\mathcal{K}_{2}^{\prime}$, but the reverse implication is not known as far as the author knows. The next proposition says that the version of $\mathcal{K}_{2}$ on our subclass of ccc forcing notions $\left(\mathcal{K}_{2}(a(\operatorname{arec}))\right)$ is equivalent to the version of $\mathcal{K}_{2}$ on partitions with the rectangle refining property $\left(\mathcal{K}_{2}(\mathrm{rec})\right)$ (which is a stronger condition of the $\mathrm{ccc}$ ).

Proposition 4.5. The following are equivalent.

(1) $\mathcal{K}_{2}(\mathrm{rec})$

(2) $\mathcal{K}_{2}(a(\operatorname{arec}))$

(3) For every forcing notion $\mathbb{P}$ with the anti-rectangle refining property, any uncountable subset of $\mathbb{P}$ has an uncountable antichain in $\mathbb{P}$. 
Proof. $(1 \Rightarrow 2)$ Assume that $\mathbb{P}$ has the anti-rectangle refining property and $I$ is a subset of $a(\mathbb{P})$ of size $\aleph_{1}$. Without loss of generality, we may assume that $I$ forms a $\Delta$-system with a root $\sigma$. Let $I^{\prime}:=\{\tau \backslash \sigma ; \tau \in I\}$. We consider the following 2-colored partition of $\left[I^{\prime}\right]^{2}$ such that for distinct $p$ and $q$ in $I^{\prime}$,

$$
\{p, q\} \in K_{0}: \Longleftrightarrow p \not \perp_{a(\mathbb{P})} q .
$$

Since $I^{\prime}$ is pairwise disjoint, it satisfies the rectangle refining property. By $\mathcal{K}_{2}(\mathrm{rec})$, there exists $J^{\prime} \in\left[I^{\prime}\right]^{\aleph_{1}}$ which is $K_{0}$-homogeneous. Let $J:=\left\{p \cup \sigma ; p \in J^{\prime}\right\}$. Then $J$ is an uncountable subset of $I$ and pairwise compatible in $a(\mathbb{P})$.

$(2 \Rightarrow 3)$ Assume that $\mathbb{P}$ satisfies the anti-rectangle refining property and $I \in$ $[\mathbb{P}]^{\aleph_{1}}$. Then the set $\{\{p\} ; p \in I\}$ is an uncountable subcollection of $a(\mathbb{P})$. By $\mathcal{K}_{2}(a(\operatorname{arec}))$, there exists $I^{\prime} \in[I]^{\aleph_{1}}$ such that the set $\left\{\{p\} ; p \in I^{\prime}\right\}$ is pairwise compatible in $a(\mathbb{P})$, i.e. for each different $p$ and $q$ in $I^{\prime}, p \perp_{\mathbb{P}} q$, i.e. $I^{\prime}$ is an uncountable antichain in $\mathbb{P}$. Let

$(3 \Rightarrow 1)$ Let $\left[\omega_{1}\right]^{2}=K_{0} \cup K_{1}$ be a partition with the rectangle refining property.

$$
\mathbb{P}:=\left\{p \in\left[\omega_{1}\right]^{<\aleph_{0}} ;[p]^{2} \subseteq K_{0}\right\},
$$

ordered by reverse inclusion. We consider the following suborder $a^{\prime \prime}(\mathbb{P})$ of $a(\mathbb{P})$ such that

$$
a^{\prime \prime}(\mathbb{P}):=\{\sigma \in a(\mathbb{P}) ; \sigma \text { is pairwise disjoint }\} .
$$

It follows from the rectangle refining property of our partition that $a^{\prime \prime}(\mathbb{P})$ has the anti-rectangle refining property. Let $I:=\left\{\{\{\alpha\}\} ; \alpha \in \omega_{1}\right\}$, which is an uncountable subset of members of $a^{\prime \prime}(\mathbb{P})$. By the property 3 , we can find $I^{\prime} \in[I]^{\aleph_{1}}$ such that $\left\{\{\{\alpha\}\} ; \alpha \in I^{\prime}\right\}$ is an antichain in $a^{\prime \prime}(\mathbb{P})$, i.e. for each different $\alpha$ and $\beta$ in $I^{\prime}$,

$$
\{\{\alpha\}\} \perp_{a^{\prime \prime}(\mathbb{P})}\{\{\beta\}\},
$$

which means that $\{\alpha\} \not \perp_{\mathbb{P}}\{\beta\}$, i.e. $\{\alpha, \beta\} \in K_{0}$. Therefore $I^{\prime}$ is an uncountable homogeneous set.

4.2. Separating $\mathcal{C}^{2}$ and $\mathrm{MA}_{\aleph_{1}}(a(\operatorname{arec}))$. In [14], Todorčević proved that $\mathcal{C}^{2}$ contradicts the existence of an entangled set of reals of size $\aleph_{1}$. A set $E$ of reals is entangled if $E$ is uncountable and for every $n \in \omega$ and every $s \in 2^{n}$ and every uncountable family $F$ of increasing (with respect to the usual ordering of the reals) pairwise disjoint $n$-tuples of elements in $E$, there exist $x$ and $y$ in $F$ such that for every $i \in n, x(i)<y(i)$ iff $s(i)=0$ (which is defined in [1, §8.]). It has been proved that MA(Productive-ccc) plus the statement that every Aronszajn tree is special does not imply MA (see in [3, Corollary 3.11.]). This follows from the following Todorčević's theorem [3, Theorem 3.10.]: It is consistent with ZFC that MA(Productive-ccc) holds, every Aronszajn tree is special and there exists an entangle set of reals. By a similar argument $^{1}$ we can show the following theorem.

\footnotetext{
${ }^{1}$ In the proof of Lemma 3.12 which is the key lemma for the proof of Theorem 3.10, there is a trivial mistake.

Let $T$ be an Aronszajn tree and $A_{0}$ and $A_{1}$ uncountable subsets of $\mathcal{Q}_{T}$ (which is just $a(T)$ in our notation). In the proof, it is shown that $a_{x} \in A_{0}$ and $b_{y} \in A_{1}$ are found such that $a_{x}$ and $b_{y}$ are compatible in $\mathcal{Q}_{T}$. But it does not hold in general. For example, let $s_{0}$ and $s_{1}$ be nodes in $T$ with $s_{0}<_{T} s_{1}$, and let $\left\langle t_{\alpha} ; \alpha \in \omega_{1}\right\rangle$ be an pairwise incomparable sequence of nodes in $T$ such that each $t_{\alpha}$ is not comparable with $s_{0}$ in $T$. Let $A_{0}:=\left\{\left\{s_{0}, t_{\alpha}\right\} ; \alpha\right.$ is odd $\}$ and $A_{1}:=\left\{\left\{s_{1}, t_{\alpha}\right\} ; \alpha\right.$ is even $\}$. Then for any $\sigma \in A_{0}$ and $\tau \in A_{1}, \sigma$ and $\tau$ are incompatible in $\mathcal{Q}_{T}$.
} 
Theorem 4.6. It is consistent with ZFC that $\mathrm{MA}(a(\operatorname{arec}))$ holds and there exists an entangled set of reals, so we conclude that $\mathrm{MA}(a(\operatorname{arec}))$ does not imply $\mathcal{C}^{2}$.

The only difference between the proof of [3, Theorem 3.10.] and Theorem 4.6 is Lemma 4.8 which is related to [3, Lemma 3.12.]. The theorem follows from the next two lemmata.

Lemma 4.7 ([3, Lemma 3.14]). The preservation of the entangledness of sets of reals by ccc forcing is preserved by finite support iterations.

Lemma 4.8. Suppose that $E$ is an entangled set of reals and $\mathbb{P}$ is a forcing notion which has the anti-rectangle refining property. Then $E$ is still entangled in the extension by $a(\mathbb{P})$.

Proof. This proof is essentially same to the proof in [3, Lemma 3.12.]. We use the following fact:

[3, Fact 3.13.]: If $E$ is an entangled set of reals, then for every $n \in \omega$, every $s \in 2^{n}$ and every uncountable family $F$ of increasing pairwise disjoint $n$-tuples of elements in $E$, there are $F_{0}$ and $F_{1}$ in $[F]^{\aleph_{1}}$ such that for every $x \in F_{0}$ and every $y \in F_{1}$ and every $i \in n, x(i)<y(i)$ iff $s(i)=0$.

Proof of this fact. By recursion on $\alpha \in \omega_{1}$, we can take $x_{\alpha}$ and $y_{\alpha}$ in $F$ and rationals $z_{\alpha, i}^{0}$ and $z_{\alpha, i}^{1}$ for each $i \in n$ such that

- $x_{\alpha}$ and $y_{\alpha}$ is in $F \backslash\left\{x_{\beta}, y_{\beta} ; \beta \in \alpha\right\}$,

- for every $i \in n, x_{\alpha}(i)<y_{\alpha}(i)$ iff $s(i)=0$, and

- if $s(i)=0$, then $x_{\alpha}(i)<z_{\alpha, i}^{0}<y_{\alpha}(i)$, and if $s(i)=1$ and $x_{\alpha}(i)>y_{\alpha}(i)$, then $x_{\alpha}(i)>z_{\alpha, i}^{1}>y_{\alpha}(i)$.

Let $I$ be an uncountable set of countable ordinals such that for every $\alpha$ and $\beta$ in $I$ and $i \in n, z_{\alpha, i}^{0}=z_{\beta, i}^{0}$ and $z_{\alpha, i}^{1}=z_{\beta, i}^{1}$, and for each $i \in n$ with $s(i)=1$ and $\alpha$ and $\beta$ in $I$,

$$
x_{\alpha}(i)=y_{\alpha}(i) \text { iff } x_{\beta}(i)=y_{\beta}(i) .
$$

For each $i \in n$ with $x_{\alpha}(i)=y_{\alpha}(i)(\alpha \in I)$, either there exists an uncountable subset $I^{\prime}$ of $I$ such that $x_{\alpha}(i)=y_{\alpha}(i)=x_{\beta}(i)=y_{\beta}(i)$, or we take a rational $w_{i}$ such that both the sets $\left\{\xi \in I ; x_{\xi}(i)>w_{i}\right\}$ and $\left\{\xi \in I ; w_{i}>y_{\xi}(i)\right\}$ are uncountable. By this observation, we can take an uncountable subset $I^{\prime}$ of $I$ such that letting $F_{0}:=\left\{x_{\xi} ; \xi \in I^{\prime}\right\}$ and $F_{1}:=\left\{y_{\xi} ; \xi \in I^{\prime}\right\}, F_{0}$ and $F_{1}$ satisfy the desired property. $\dashv$

Let $n \in \omega, s \in 2^{n}, \dot{F}$ be a $a(\mathbb{P})$-name for an uncountable family of increasing pairwise disjoint $n$-tuples of elements in $E$, and $p \in a(\mathbb{P})$. (We should notice that the set of finite subsets of $E$ in the extension is same to one in the ground model.) We can find a pair $\left\langle p_{\xi}, x_{\xi}\right\rangle$, for $\xi \in \omega_{1}$, of a condition below $p$ in $a(\mathbb{P})$ and an $n$-tuples of elements in $E$ such that

$$
p_{\xi} \Vdash_{a(\mathbb{P})} " \check{x_{\xi}} \in \dot{F} "
$$

and the family $\left\{x_{\xi} ; \xi \in \omega_{1}\right\}$ is pairwise disjoint. Without loss of generality, we may assume that the set $\left\{p_{\xi} ; \xi \in \omega_{1}\right\}$ forms a $\Delta$-system with root $r$. By the above fact, there are $I_{0}$ and $I_{1}$ in $\left[\omega_{1}\right]^{\aleph_{1}}$ such that for all $\xi \in I_{0}, \eta \in I_{1}$ and $i \in \omega$, $x_{\xi}(i)<x_{\eta}(i)$ iff $s(i)=0$. Applying Proposition 2.2 to the set $\left\{p_{\xi} \backslash r ; \xi \in I_{0}\right\}$ and $\left\{p_{\xi} \backslash r ; \xi \in I_{1}\right\}$, we can find $I_{0}^{\prime} \in\left[I_{0}\right]^{\aleph_{1}}$ and $I_{1}^{\prime} \in\left[I_{1}\right]^{\aleph_{1}}$ such that for every $\xi \in I_{0}^{\prime}$ 
and $\eta \in I_{1}^{\prime}, p_{\xi}$ and $p_{\eta}$ are compatible in $a(\mathbb{P})$. Then for some (any) $\xi \in I_{0}^{\prime}$ and $\eta \in I_{1}^{\prime}, p_{\xi} \cup p_{\eta}$ is a common extension of $p_{\xi}$ and $p_{\eta}$ and

$$
p_{\xi} \cup p_{\eta} \Vdash_{a(\mathbb{P})} “ \check{x_{\xi}}, \check{x_{\eta}} \in \dot{F} "
$$

and $x_{\xi}(i)<x_{\eta}(i)$ iff $s(i)=0$, for all $i \in n$, which finishes the proof.

In [10], Kunen-Tall proved that it is consistent with ZFC that $\mathrm{MA}_{\aleph_{1}}$ (property $\mathrm{K}$ ) holds and Suslin's Hypothesis fails (i.e. there exists a Suslin tree). So $M A_{\aleph_{1}}$ and $\mathrm{MA}_{\aleph_{1}}$ (property K) can be separated. Therefore we have the following diagram:

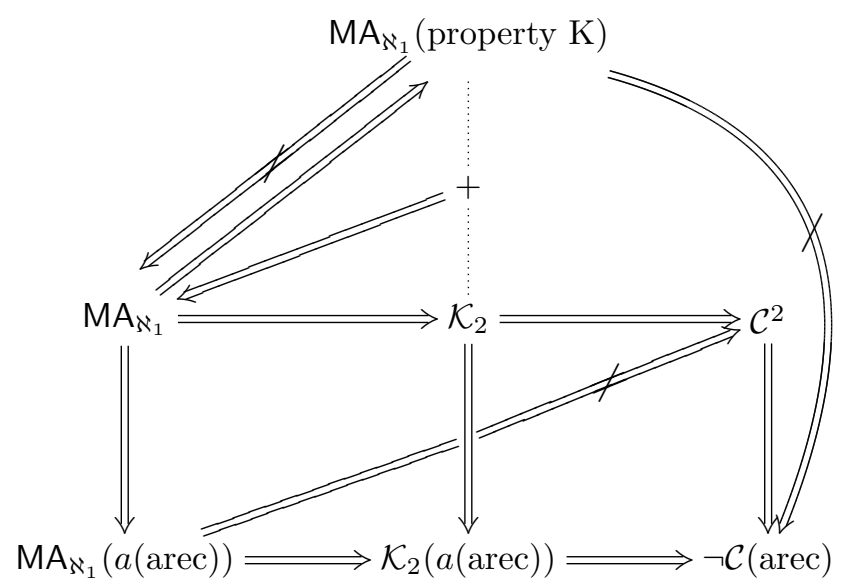

Acknowledgements. I would like to thank Sakaé Fuchino, Daisuke Ikegami, Masaru Kada and Yasuo Yoshinobu for useful comments, and Ilijas Farah, Paul B. Larson and Justin Moore for useful conversations about Todorčević's works on fragments of Martin's Axiom.

I would also like to thank the referee for kind comments.

\section{REFERENCES}

[1] U. Abraham, M. Rubin and S. Shelah. On the consistency of some partition theorems for continuous colorings, and the structure of $\aleph_{1}$-dense real order types, Ann. Pure Appl. Logic 29 (1985), no. 2, 123-206.

[2] U. Abraham and S. Todorčević. Partition properties of $\omega_{1}$ compatible with CH, Fund. Math. 152 (1997), 165-180.

[3] J. Bagaria. Fragments of Martin's axiom and $\Delta_{3}^{1}$ sets of reals, Ann. Pure Appl. Logic 69 (1994), no. 1, 1-25.

[4] J. Baumgartner, J. Malitz and W. Reinhardt. Embedding trees in the rationals, Proc. Nat. Acad. Sci. U.S.A. 67 (1970), 1748-1753.

[5] H. Dales and H. Woodin. An introduction to independence for analysts, London Mathematical Society Lecture Note Series, 115.

[6] T. Jech. Set theory. The third millennium edition, revised and expanded, Springer Monographs in Mathematics, Springer-Verlag, Berlin, 2003.

[7] S. Kamo. Almost coinciding families and gaps in $\mathcal{P}(\omega)$, J. Math. Soc. Japan 45 (1993), no. $2,357-368$.

[8] B. Knaster. Sur une propriété caractéristique de l'ensemble des nombres réela, Mat. Sb. 16 (1945), 281-288.

[9] K. Kunen. $\left(\kappa, \lambda^{*}\right)$-gaps under MA, handwritten note, 1976.

[10] K. Kunen and F. Tall. Between Martin's axiom and Souslin's hypothesis, Fund. Math. 102 (1979), no. 3, 173-181.

[11] P. Larson and S. Todorčević. Chain conditions in maximal models, Fund. Math. 168 (2001), no. 1, 77-104. 
[12] P. Larson and S. Todorčević. Katětov's problem, Trans. Amer. Math. Soc. 354 (2002), no. $5,1783-1791$

[13] M. Scheepers. Gaps in $\omega^{\omega}$, In Set Theory of the Reals, volume 6 of Israel Mathematical Conference Proceedings, 439-561, 1993.

[14] S. Todorčević. Remarks on chain conditions in products, Compositio Math. 55 (1985), no. 3, 295-302.

[15] S. Todorčević and B. Veličiković. Martin's axiom and partitions, Compositio Math. 63 (1987), no. 3, 391-408

[16] S. Todorčević. Partition Problems in Topology, volume 84 of Contemporary mathematics, American Mathematical Society, Providence, Rhode Island, 1989.

[17] T. Yorioka. The diamond principle for the uniformity of the meager ideal implies the existence of a destructible gap, Arch. Math. Logic 44 (2005), 677-683. 